\title{
Mixed Platoon Flow Dispersion Model Based on Speed-Truncated Gaussian Mixture Distribution
}

\author{
Weitiao Wu, Wenzhou Jin, and Luou Shen \\ School of Civil and Transportation Engineering, South China University of Technology, Guangzhou 510641, China \\ Correspondence should be addressed to Luou Shen; ctwshen@163.com
}

Received 13 March 2013; Revised 11 May 2013; Accepted 11 May 2013

Academic Editor: Shuyu Sun

Copyright (C) 2013 Weitiao Wu et al. This is an open access article distributed under the Creative Commons Attribution License, which permits unrestricted use, distribution, and reproduction in any medium, provided the original work is properly cited.

\begin{abstract}
A mixed traffic flow feature is presented on urban arterials in China due to a large amount of buses. Based on field data, a macroscopic mixed platoon flow dispersion model (MPFDM) was proposed to simulate the platoon dispersion process along the road section between two adjacent intersections from the flow view. More close to field observation, truncated Gaussian mixture distribution was adopted as the speed density distribution for mixed platoon. Expectation maximum (EM) algorithm was used for parameters estimation. The relationship between the arriving flow distribution at downstream intersection and the departing flow distribution at upstream intersection was investigated using the proposed model. Comparison analysis using virtual flow data was performed between the Robertson model and the MPFDM. The results confirmed the validity of the proposed model.
\end{abstract}

\section{Introduction}

Traffic flow in urban areas presents interrupted flow features. Due to the compression and splitting by signal lights, traffic flow is separated into series and moves downstream in platoons. Vehicles in platoon travel at different speeds because of the diverse behaviors of drivers and maneuvering characteristics of vehicles. While moving downstream, the platoon starts spreading in a longer segment which is called platoon dispersion. Platoon dispersion modeling is one of the key aspects in intelligent transportation system (ITS) area, which provides theoretical support for signal coordination control.

Many researchers have worked on the platoon dispersion topic. Pacey [1] first studied the diffusion problem and proposed a model assuming that the speed follows normal distribution ranging from negative to positive infinity. Grace and Potts [2] further investigated Pacey's model from the density view. Robertson [3], using data collected by Hillier and Rothery [4], developed a recurrent dispersion model that is widely used in signal coordination optimization and control systems such as TRANSYT [5], SCOOT [6], SATURN [7], and TRAFLO [8]. Seddon [9] found that Robertson's model was based on travel time shifted geometric distribution. Tracz [10] and Polus [11] have shown that vehicular travel time distribution is not necessarily a shifted geometric distribution as in Robertson's model and is more consistent with a normal, lognormal or a gamma distribution. Liu and Yang [12-14] studied Grace's model using field data collected in Shanghai, China, proposed a method to correct the vehicle startup time loss, and analyzed the problem of the front and rear of the platoon. Wang et al. $[15,16]$ developed a platoon dispersion model under the assumption that the travel time follows normal distribution and found it to be a better-fitted field data than Pacey's assumption. Wei et al. [17] proposed a platoon dispersion model for cars from the density view assuming speed following truncated normal distribution.

Robertson's model in TRANSYT implies dispersion by the platoon dispersion factor for three external friction levels. Manar and Baass [18] demonstrate that platoon dispersion depends not only on external friction but also on internal friction measured by volume and density and developed mathematical models relating platoon dispersion to internal and external frictions. G. C. K. Wong and S. C. Wong [19] developed a multiclass traffic flow model as an extension of the LWR model, which considered the heterogeneous 
drivers. Bonneson et al. [20] developed a procedure for prediction of the arrival flow profile for an intersection approach considering platoon decay due to mid-segment driveway access and egress, which tends to have a significant impact on the arrival flow profile. In a recent study, Cheng [21] found that the traffic flow on China urban roads presents a characteristic of mixed vehicle speed distributions. Chen et al. [22] analyzed the bus-car mixed traffic, and results show that the bus ration has significant impact on the speed distribution.

The literature review shows that researchers have doubted the distribution assumptions of both Pacey's and Robertson's models. However, due to the simplicity of Robertson's recurrent equation, it has received the most popularity. Meanwhile, very few studies tried to develop a new dispersion model. Recent researches present a trend investigating the impact of heterogeneity, mixed flow, and internal frictions on platoon dispersion.

The traffic on urban arterials in China presents a mixed flow feature due to the large amount of buses. Typically, buses run on three types of facilities: normal lanes with mixed traffic, dedicated bus lanes, and bus rapid traffic (BRT) lanes.

Dedicated bus lanes and BRT lanes are special lanes separated from other traffic by road markings or physical barriers, which present unique operational features. However, urban arterials in China mostly belong to the first class, which present mixed traffic flow.

Generally, the percentage of buses in mixed traffic flow varies from $10 \%$ to $25 \%$ during peak periods. Mixed platoon presents special characteristics compared to car platoon because of the lesser maneuverability of buses and the running speed constrained by scheduled stops. Previous research has not been done on bus platoon dispersion modeling, and no car and bus mixed platoon dispersion model has been developed either. The investigation of the mixed platoon dispersion problem will provide theoretical support for signal coordination and bus priority control.

\section{Model Development}

2.1. Speed Density Distribution Assumption. In Pacey's platoon dispersion model, the speed is assumed following normal distribution ranging from negative to positive infinity, which does not properly reflect the field situation. Because vehicles with speeds $v<v_{\min }$ and $v>v_{\max }\left(v_{\min }\right.$ and $v_{\max }$ denote minimum speed and maximum speed, resp.) are rarely observed in the actual world, which is confirmed by field data as shown in the data acquisition and analysis section given below, therefore, the assumed speed following truncated distribution ranging from $v_{\min }$ to $v_{\max }$ is more suitable. The distribution can be truncated normal distribution or other. In this study, due to the fact that the field data fits the truncated Gaussian mixture distribution (TGMD) better and its widely use with simple mathematic form, the TGMD is chosen to demonstrate the development of the mixed platoon dispersion model based on speed-truncated distribution.
By modifying Pacey's speed normal distribution, the proposed TGMD is shown in the following equation:

$$
f(v)= \begin{cases}\sum_{j=1}^{M} \alpha_{j} p_{j}\left(v \mid \mu_{j}, \sigma_{j}\right)=c \sum_{j=1}^{M} \alpha_{j} \frac{1}{\sqrt{2 \pi} \sigma_{j}} e^{-0.5\left(\left(v-\mu_{j}\right) / \sigma_{j}\right)^{2}}, \\ v_{\min } \leq v \leq v_{\max }, \sum_{j=1}^{M} \alpha_{j}=1, \\ \text { others, }\end{cases}
$$

where, $\alpha_{j}, \mu_{j}$, and $\sigma_{j}, j=1,2 \ldots, M$, are the parameters of Gaussian mixture distribution, which can be estimated by EM algorithm [23, 24], $M$ is the number of mixed component, and $c$ is a parameter ensuring that the accumulated probability of $f(v)$ in range $\left[v_{\min }, v_{\max }\right]$ equals $100 \%$. As for $v_{\min } \leq v \leq v_{\max }$, because $\int_{v_{\min }}^{v_{\max }} f(v) d v=1$, then $c^{-1}=$ $\sum_{j=1}^{M} \alpha_{j}\left[\Phi\left(v_{\max } / \sigma_{j}-\mu_{j} / \sigma_{j}\right)-\Phi\left(v_{\min } / \sigma_{j}-\mu_{j} / \sigma_{j}\right)\right]$, where $\Phi$ denotes the cumulative function of the standard normal distribution.

2.2. Platoon Flow Dispersion Model. Assuming the start time of the green phase of upstream signal $t=0$ and the stop bar location $x=0$, then, the departing flow function when the upstream intersection signal turns to green is $q^{\prime}(x=0, t)$. For signal coordination control, the arriving flow distribution downstream is used to calculate parameters such as delay, stop, and queue length based on shock wave theory. Therefore, it is important to develop a model to predict the arriving flow function from the upstream departing flow function. The following section presents the model development process.

During time differential $[T, T+d T]$, the departing vehicles from the upstream intersection stop line are $q^{\prime}(x=$ $0, T) d T$; following speed-truncated distribution assumption, the vehicle flow $q^{\prime}(x=0, T=t-x / v) f(v) d v$ leaving at time $t-x / v$ from the upstream intersection stop line will arrive at the downstream intersection $x(x>0)$ at time $t$, which is $q^{\prime}(x=0, T) f(v) d v d T$. Therefore, the number of vehicles arriving at downstream intersection during time differential $[t, t+d t]$ can be expressed using the following integration equation:

$$
q(x, t) d t=\int_{T_{1}=t-x / v_{1}}^{T_{2}=t-x / v_{2}} f(v) q\left(x=0, T=t-\frac{x}{v}\right) d v d T .
$$

Then, after dividing by the time differential in both sides of (2), the arriving flow rate at downstream intersection becomes

$$
q(x, t)=\int_{v_{1}}^{v_{2}} f(v) q\left(x=0, T=t-\frac{x}{v}\right) d v,
$$

where $v_{1}$ and $v_{2}$ represent the minimum and maximum speeds for those vehicles arriving at downstream location $x$ at time $t$.

Without loss of generality, there are three typical departing flow patterns in the actual world: stable linear flow, 
decreasing linear flow, and stable combined with decreasing linear flow as demonstrated in Figure 1. The following section will develop the arriving flow function at the downstream intersection based on speed TGMD assumption.

2.2.1. Stable Linear Flow Pattern. The departing flow function of the stable linear flow pattern at the upstream intersection stop line $x=0$ is $q^{\prime}(x=0, T)$, which can be expressed in the following equation:

$$
q^{\prime}(x=0, T)= \begin{cases}q, & 0<T \leq g \\ 0, & T>g\end{cases}
$$

where $g$ is the duration of the green phase and $q$ is the departing saturation flow rate.

Then, the arriving flow function at downstream intersection location $x$ at time $t$ can be expressed as the following piecewise function:

(a) when $x / v_{\min } \leq x / v_{\max }+g$,

$$
q(x, t)= \begin{cases}0, & t<\frac{x}{v_{\max }} \cup t>\frac{x}{v_{\min }}+g \\ q \int_{x / t}^{v_{\max }} f(v) d v, & \frac{x}{v_{\max }} \leq t<\frac{x}{v_{\min }} \\ q \int_{v_{\operatorname{mix}}}^{v_{\max }}(v) d v, & \frac{x}{v_{\min }} \leq t \leq \frac{x}{v_{\max }}+g \\ q \int_{v_{\min }}^{x /(t-g)} f(v) d v, & \frac{x}{v_{\max }}+g<t \leq \frac{x}{v_{\min }}+g,\end{cases}
$$

(b) when $x / v_{\min }>x / v_{\max }+g$,

$$
q(x, t)= \begin{cases}0, & t<\frac{x}{v_{\max }} \cup t>\frac{x}{v_{\min }}+g \\ q \int_{x / t}^{v_{\max }} f(v) d v, & \frac{x}{v_{\max }} \leq t<\frac{x}{v_{\max }}+g \\ q \int_{x / t}^{x /(t-g)} f(v) d v, & \frac{x}{v_{\max }}+g \leq t \leq \frac{x}{v_{\min }} \\ q \int_{v_{\min }}^{x /(t-g)} f(v) d v, & \frac{x}{v_{\min }}<t \leq \frac{x}{v_{\min }}+g .\end{cases}
$$

Let $u=(v-\mu) / \sigma$ and the dispersion rate $\alpha=\sigma / \mu$, because

$$
\begin{aligned}
\int_{v_{1}}^{v_{2}} f & (v) d v \\
= & c \sum_{j=1}^{M} \alpha_{j} \int_{v_{1}}^{v_{2}} \frac{1}{\sqrt{2 \pi} \sigma_{j}} e^{-0.5\left(\left(v-\mu_{j}\right) / \sigma_{j}\right)^{2}} d v \\
& =c \sum_{j=1}^{M} \alpha_{j} \int_{\left(v_{1}-\mu_{j}\right) / \sigma_{j}}^{\left(v_{2}-\mu_{j}\right) / \sigma_{j}} \frac{1}{\sqrt{2 \pi}} e^{-0.5 u^{2}} d u \\
& =c \sum_{j=1}^{M} \alpha_{j} \int_{\left(t v_{1} / \mu_{j}-t\right) / \alpha_{j} t}^{\left(t v_{2} / \mu_{j}-t\right) / \alpha_{j} t} \frac{1}{\sqrt{2 \pi}} e^{-0.5 u^{2}} d u,
\end{aligned}
$$

$$
\begin{aligned}
& =\frac{c}{2} \sum_{j=1}^{M} \alpha_{j}\left(2 \int_{0}^{\left(t v_{2} / \mu_{j}-t\right) / \sqrt{2} \alpha_{j} t} \frac{1}{\sqrt{2 \pi}} e^{-0.5 u^{2}} d u\right. \\
& \left.\quad-2 \int_{0}^{\left(t v_{1} / \mu_{j}-t\right) / \sqrt{2} \alpha_{j} t} \frac{1}{\sqrt{2 \pi}} e^{-0.5 u^{2}} d u\right) \\
& =\frac{c}{2} \sum_{j=1}^{M} \alpha_{j}[\Phi(z)]_{z_{j, 1}}^{z_{j, 2}},
\end{aligned}
$$

where $z_{j, 2}=\left(t v_{2} / \mu_{j}-t\right) / \sqrt{2} \alpha_{j} t, z_{j, 1}=\left(t v_{1} / \mu_{j}-\right.$ t) $/ \sqrt{2} \alpha_{j} t$, and $v_{1}$ and $v_{2}$ are constants, because $\Phi(z)=$ $2 \int_{0}^{\sqrt{2} z}(1 / \sqrt{2 \pi}) e^{-0.5 u^{2}} d u=(2 / \sqrt{\pi}) \int_{0}^{z} e^{-u^{2}} d u$ is the accumulated probability function of standard normal distribution. Based on (5), (6), and (7), $q(x, t)$ can be calculated using the following formula:

(a) when $x / v_{\min } \leq x / v_{\max }+g$,

$q(x, t)$

$$
\left\{\begin{array}{c}
0, \quad t>\frac{x}{v_{\max }} \cup t<\frac{x}{v_{\min }}+g \\
\left.\frac{c q}{2} \sum_{j=1}^{M} \alpha_{j}[\Phi(z)]\right]_{\left(v_{\max } / \mu_{j}-t\right) / \sqrt{2} \alpha_{j} t}^{\left(t / \mu_{j}-t\right) / \sqrt{2} \alpha_{j} t}, \\
\frac{x}{v_{\max }} \leq t<\frac{x}{v_{\min }} \\
\left.\frac{c q}{2} \sum_{j=1}^{M} \alpha_{j}[\Phi(z)]\right]_{\left(t v_{\min } / \mu_{j}-t\right) / \sqrt{2} \alpha_{j} t^{\prime}}^{\left(t v_{\max } / \mu_{j}-t\right) / \sqrt{2} \alpha_{1} t} \\
\frac{x}{v_{\min }} \leq t \leq \frac{x}{v_{\max }}+g \\
\frac{c q}{2} \sum_{j=1}^{M} \alpha_{j}[\Phi(z)] \frac{x}{\left(t v_{\min } / \mu_{j}-t\right) / \sqrt{2} \alpha_{j} t}, \\
\frac{x}{v_{\max }}+g<t \leq \frac{x}{v_{\min }}+g,
\end{array}\right.
$$

(b) when $x / v_{\min }>x / v_{\max }+g$,

$q(x, t)$

$$
=\left\{\begin{array}{c}
0, \quad t>\frac{x}{v_{\max }} \cup t<\frac{x}{v_{\min }}+g \\
\frac{c q}{2} \sum_{j=1}^{M} \alpha_{j}[\Phi(z)]_{\left(x / \mu_{j}-t\right) / \sqrt{2} \alpha_{j} t}^{\left(t v_{\max } / \mu_{j}-t\right) / \sqrt{2} \alpha_{j} t}, \\
\frac{x}{v_{\max }} \leq t<\frac{x}{v_{\max }}+g \\
\frac{c q}{2} \sum_{j=1}^{M} \alpha_{j}[\Phi(z)]_{\left(x / \mu_{j}-t\right) / \sqrt{2} \alpha_{j} t}^{\left(t x / \mu_{j}(t-g) / \sqrt{2} \alpha_{j} t\right.}, \\
\frac{x}{v_{\max }}+g \leq t \leq \frac{x}{v_{\min }} \\
\left.\frac{c q}{2} \sum_{j=1}^{M} \alpha_{j}[\Phi(z)]\right]_{\left(t v_{\min } / \mu_{j}-t\right) / \sqrt{2} \alpha_{j} t}^{\left(t / \mu_{j}(t-g) t \sqrt{2} \alpha_{j} t\right.}, \\
\frac{x}{v_{\min }}<t \leq \frac{x}{v_{\min }}+g .
\end{array}\right.
$$




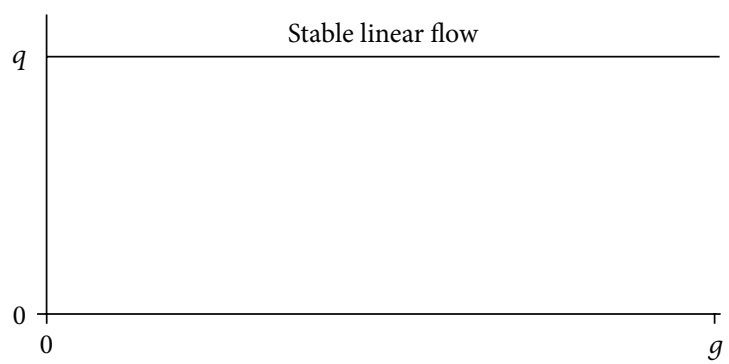

(a)

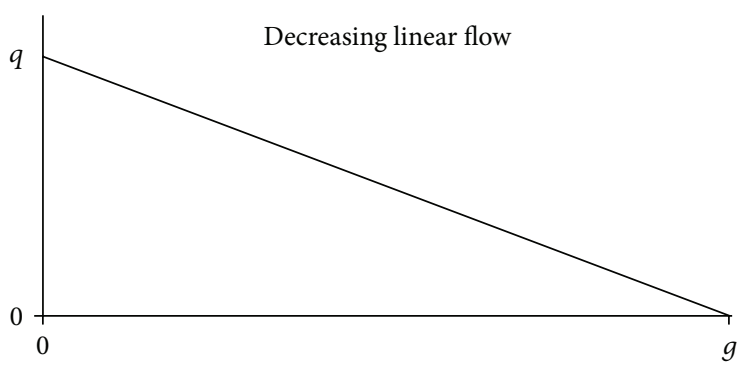

(b)

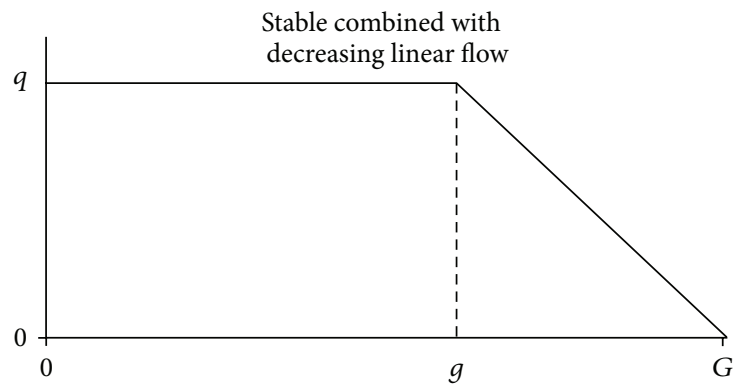

(c)

FIGURE 1: Three typical departing flow patterns.

2.2.2. Decreasing Linear Flow Pattern. The departing flow function of the decreasing linear flow pattern at the upstream intersection stop line $x=0$ during the green phase is $q^{\prime}(x=$ $0, T)$ as expressed in the following equation:

$$
q^{\prime}(x=0, T)= \begin{cases}q-a T, & 0<T \leq g \\ 0, & T>g,\end{cases}
$$

where $a=q / g$ is the linear decreasing rate.

Following the method for stable linear flow pattern, the arriving flow function at the downstream intersection location $x$ at time $t$ can be expressed as the following piecewise function:

(a) when $x / v_{\min } \leq x / v_{\max }+g$,

$$
q(x, t) \quad\left\{\begin{array}{r}
0, \\
t<\frac{x}{v_{\max }} \cup t>\frac{x}{v_{\min }}+g \\
(q-a t) \int_{x / t}^{v_{\max }} f(v) d v-a x \int_{x / t}^{v_{\max }} \frac{f(v)}{v} d v \\
\frac{x}{v_{\max }} \leq t<\frac{x}{v_{\min }} \\
(q-a t) \int_{v_{\min }}^{v_{\max }} f(v) d v-a x \int_{v_{\min }}^{\frac{f(v)}{v}} d v \\
\frac{x}{v_{\min }} \leq t \leq \frac{x}{v_{\max }}+g \\
(q-a t) \int_{v_{\min }}^{x /(t-g)} f(v) d v-a x \int_{v_{\min }}^{x /(t-g)} \frac{x(v)}{v} d v, \\
\frac{x}{v_{\max }}+g<t \leq \frac{x}{v_{\min }}+g
\end{array}\right.
$$

(b) when $x / v_{\min }>x / v_{\max }+g$,

$q(x, t)$

$$
\left\{\begin{array}{c}
t>\frac{x}{v_{\max }} \cup t<\frac{x}{v_{\min }}+g \\
(q-a t) \int_{x / t}^{v_{\max }} f(v) d v-a x \int_{x / t}^{v_{\max }} \frac{f(v)}{v} d v, \\
\frac{x}{v_{\max }} \leq t<\frac{x}{v_{\max }}+g \\
(q-a t) \int_{x / t}^{x /(t-g)} f(v) d v-a x \int_{x / t}^{x /(t-g)} \frac{f(v)}{v} d v, \\
\frac{x}{v_{\max }}+g \leq t \leq \frac{x}{v_{\min }} \\
(q-a t) \int_{v_{\min }}^{x /(t-g)} f(v) d v-a x \int_{v_{\min }}^{x /(t-g)} \frac{f(v)}{v} d v, \\
\frac{x}{v_{\min }}<t \leq \frac{x}{v_{\min }}+g .
\end{array}\right.
$$
be revised as follows: 
(a) when $x / v_{\min } \leq x / v_{\max }+g$,

$$
\begin{aligned}
& q(x, t) \\
& \left\{\begin{array}{c}
0, \quad t>\frac{x}{v_{\max }} \cup t<\frac{x}{v_{\min }}+g \\
\left.\frac{c(q-a t)}{2} \sum_{j=1}^{M} \alpha_{j}[\Phi(z)]\right]_{\left(x / \mu_{j}-t\right) / \sqrt{2} \alpha_{j} t}^{\left(t v_{\max } / \mu_{j}-t\right) / \sqrt{2} \alpha_{j} t} \\
-a x \int_{x / t}^{v_{\max }} \frac{f(v)}{v} d v, \\
\frac{x}{v_{\max }} \leq t<\frac{x}{v_{\min }} \\
\left.\frac{c(q-a t)}{2} \sum_{j=1}^{M} \alpha_{j}[\Phi(z)]\right]_{\left(x / \mu_{j}-t\right) / \sqrt{2} \alpha_{j} t}^{\left(t x / \mu_{j}(t-g) / \sqrt{2} \alpha_{j} t\right.} \\
-a x \int_{x / t}^{x /(t-g)} \frac{f(v)}{v} d v, \\
\frac{x}{v_{\min }} \leq t \leq \frac{x}{v_{\max }}+g \\
-a x \int_{v_{\min }}^{x /(t-g)} \frac{f(v)}{v} d v, \\
\frac{x}{v_{\max }}+g<t \leq \frac{x}{v_{\min }}+g,
\end{array}\right.
\end{aligned}
$$

(b) when $x / v_{\min }>x / v_{\max }+g$,

$q(x, t)$

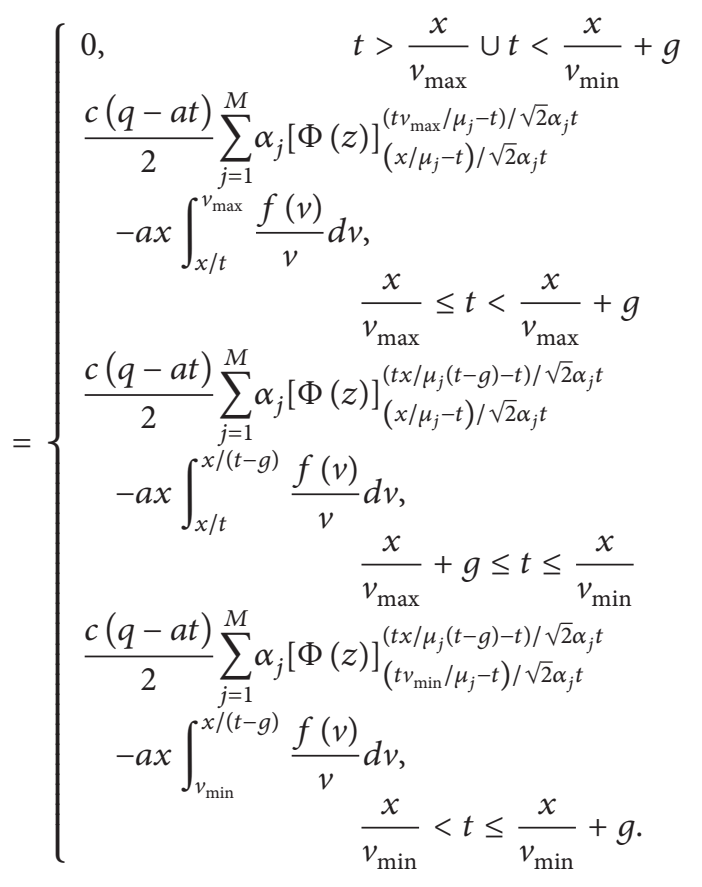

The first item in (13) and (14) can be calculated using the accumulated probability function of standard normal distribution; the second item cannot be computed by integration. Therefore, let $u=(v-\mu) / \sigma$, and the natural exponential function can be expanded applying the Taylor series as shown in the following:

$$
\begin{aligned}
& \int_{v_{1}}^{v_{2}} \frac{f(v)}{v} d v \\
& =c \sum_{j=1}^{M} \alpha_{j} \int_{\left(v_{1}-\mu_{j}\right) / \sigma_{j}}^{\left(v_{2}-\mu_{j}\right) / \sigma_{j}} \frac{1}{\sqrt{2 \pi}\left(u \sigma_{j}+\mu_{j}\right)} e^{-0.5 u^{2}} d u \\
& =c \sum_{j=1}^{M} \alpha_{j} \int_{\left(v_{1}-\mu_{j}\right) / \sigma_{j}}^{\left(v_{2}-\mu_{j}\right) / \sigma_{j}} \frac{1}{\sqrt{2 \pi}\left(u \sigma_{j}+\mu_{j}\right)} \\
& \quad \times\left(1-\frac{u^{2}}{2}+\frac{u^{4}}{8} \cdots+(-1)^{n} \frac{u^{2 n}}{n ! 2^{n}}\right) d u .
\end{aligned}
$$

The expanded Taylor series can be computed by integration. As the Taylor series method is an approximation method, for application requiring high computation accuracy the numerical integration method is needed which can be easily obtained with the help of a modern computer.

\subsubsection{Stable Combined with Decreasing Linear Flow Pattern.} The departing flow function of the stable combined with decreasing linear flow pattern at the upstream intersection stop line $x=0$ during the green phase is $q^{\prime}(x=0, T)$ as expressed in the following equation:

$$
q^{\prime}(x=0, T)= \begin{cases}q, & 0<T \leq g \\ q-a(T-g), & g<T \leq G,\end{cases}
$$

where $a=q /(G-g)$ is the linear decreasing rate.

Because different classes of flows are addable, the arriving flow function at downstream intersection for the stable combined with decreasing linear flow pattern can be expressed by adding the arriving flow of the stable linear flow pattern with the arriving flow of the decreasing linear flow pattern by shifting time $g$. The details of the calculation formula are not presented here.

The proposed MPFDM is developed as given in the previous section. If $x$ is set as the downstream signal locations, the platoon dispersion process between the two signals can be quantitatively analyzed using the model. The results can be used to calculate signal timing parameters such as delay, stop, and queue length for signal coordination and bus priority control.

\section{Data Acquisition and Analysis}

Field data were collected for model development and validation. The surveyed road is a typical four-lane two-way urban arterial, Wushan Road, which normally operates at undersaturation traffic condition. Along this road, there are 14 bus lines, and the posted speed limit is $50 \mathrm{~km} / \mathrm{h}$ $(13.89 \mathrm{~m} / \mathrm{s})$. License plates were recorded by video cameras at two locations (650 $\mathrm{m}$ distance): one is right after the signals at Yuehan Road and the other is located right before the diverging points. Travel times were directly computed from video records, and the original speeds (journey speeds for 
TABLE 1: Origin platoon journey speed data.

\begin{tabular}{|c|c|c|c|c|c|c|c|c|c|}
\hline & \multicolumn{3}{|c|}{ Period $1(7: 45-8: 25$ am $)$} & \multicolumn{3}{|c|}{ Period 2 (8:25-10:00 am) } & \multicolumn{3}{|c|}{ Period 3 (10:00-10:40 am) } \\
\hline & Car & Bus & Mixed & Car & Bus & Mixed & Car & Bus & Mixed \\
\hline Length (m) & & 650 & & & 650 & & & 650 & \\
\hline Flow rate (veh/h) & & 1007 & & & 881 & & & 648 & \\
\hline Bus traffic $(\%)$ & & 13.1 & & & 10.8 & & & 11.8 & \\
\hline Sample size & 617 & 88 & 705 & 1244 & 151 & 1395 & 410 & 51 & 461 \\
\hline Minimum speed (m/s) & 8.67 & 5.65 & 5.65 & 7.74 & 5.37 & 5.37 & 7.22 & 6.13 & 6.13 \\
\hline Maximum speed (m/s) & 20.97 & 14.44 & 20.97 & 21.67 & 12.50 & 21.67 & 20.96 & 13.27 & 20.96 \\
\hline Average speed (m/s) & 13.52 & 7.80 & 12.85 & 13.62 & 8.14 & 13.23 & 13.97 & 8.10 & 13.31 \\
\hline Standard deviation & 1.99 & 1.79 & 2.64 & 1.90 & 1.34 & 2.56 & 1.85 & 1.61 & 2.44 \\
\hline
\end{tabular}

TABLE 2: Estimated parameter values of Gaussian mixture distribution based on EM algorithm.

\begin{tabular}{|c|c|c|c|c|c|c|c|}
\hline & \multicolumn{3}{|c|}{ 1st component } & \multicolumn{3}{|c|}{ 2nd component } & \multirow{2}{*}{ Iteration times } \\
\hline & $\alpha_{1}$ & $\mu_{1}$ & $\sigma_{1}$ & $\alpha_{2}$ & $\mu_{2}$ & $\sigma_{2}$ & \\
\hline Period 1 & 0.8290 & 13.6642 & 3.2344 & 0.1710 & 8.9297 & 4.0870 & 3 \\
\hline Period 2 & 0.9065 & 13.5757 & 4.1019 & 0.0935 & 7.6659 & 0.8087 & 22 \\
\hline Period 3 & 0.9004 & 13.9452 & 3.6320 & 0.0996 & 7.5916 & 0.4910 & 7 \\
\hline
\end{tabular}

buses, running speed for cars) were derived from travel time and distance. The data was collected from 7:45 AM to $10: 40$ AM. Three time periods were apparently identified based on different traffic volume levels. A statistics summary of the original car and mixed platoon speed data for all time periods is presented in Table 1 .

As shown in Table 1, the average speeds of the car platoon are slightly higher than those of mixed platoon for all time periods; the standard deviations of car platoon are lower than those of mixed platoon; the minimum speeds of car platoon are greater than those of mixed platoon; the maximum speeds of car platoon are the same as those of mixed platoon. All these are reasonable because buses have lower speed compared to cars presented in the mixed platoon.

The plots of the speed histogram and the fitted Gaussian mixture distribution curves are shown in Figure 2. From the plots, two humps were obviously identified from the speed data histogram of the mixed platoon, which represent car and bus groups, respectively. This confirmed the results of Cheng [21] and Chen et al. [22]. For this reason, some researchers $[25,26]$ have proposed to use compound distributions that use an appropriate combination of more than one distribution as a modeling tool, since the fitting of corresponding distributions is usually regarded as the "dissection" of a heterogeneous population into more homogeneous "parts." Due to the fact that Gaussian mixture distribution can approximate any continuous distribution and its widely use with simple mathematic form composed of several weighted normal distributions, Gaussian mixture distribution is used in this paper. Let the number of mixed component $M=$ 2, based on MATLAB software, and parameters for all time periods were obtained using EM algorithm as listed in Table 2. Because EM algorithm is widely known, its details are not presented here in order to keep conciseness.
Furthermore, performances of different distributions (including normal, lognormal, Weibull, and gamma) fitting for the mixed platoon speed data present K-S evaluation $P$ values $<0.01$ with 0.05 of significance level due to different speed distribution characteristics of cars and buses in the mixed platoon. Nevertheless, Gaussian mixture distribution is the one with K-S evaluation $P$ values $>0.15$ for all time periods. Because the speed of Gaussian mixture distribution spreads within a limited value range between minimum speed and maximum speed, we can accept the assumption that speed follows truncated Gaussian mixture distribution, which is composed of several components of truncated normal distribution with the same range limit $[25,26]$.

What is worth mentioning is that $M$ is usually determined using the histogram observation method, whose detailed steps are to draw the envelope of sample data histogram and observe the number of curve peaks $F$, generally required $F \leq$ $M<2 F$.

\section{Platoon Flow Dispersion Analysis}

Because the MPFDM demonstrated here assumes the speed following TGMD, the parameters used in the model need to be transferred from the Gaussian mixture distribution. The TGMD statistics of the vehicle speed data and the parameters estimated by EM algorithm of period 1 are summarized in Table 3, which is used in this section to demonstrate the application of MPFDM in signal coordination analysis.

4.1. Departing Flow Function at Upstream Intersection. To compare the performance of the proposed model with the Robertson model, virtual departing flow distributions for mixed platoon from upstream intersection are assumed for 


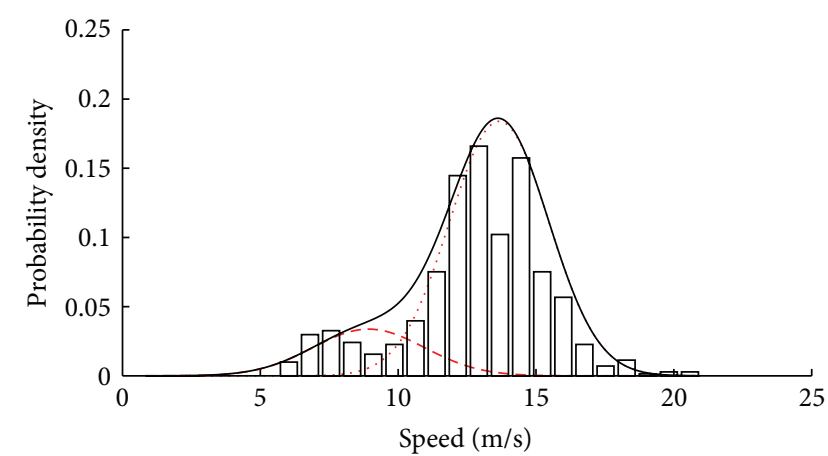

$\begin{array}{lll}\ldots . . . & \text { 1st component curve } \quad \square & \text { Fitted Gaussian mixture curve } \\ --- \text { 2nd component curve } \square \quad \text { Frequency histogram }\end{array}$

(a) Period 1

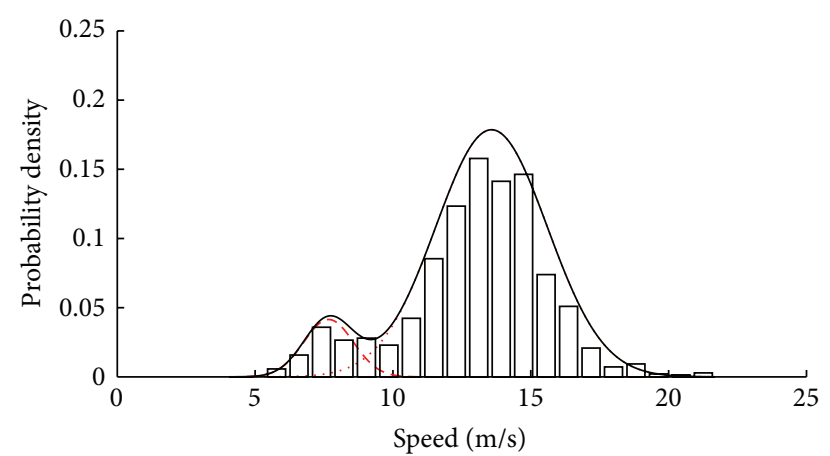

…. 1st component curve - Fitted Gaussian mixture curve --- 2nd component curve $\square$ Frequency histogram

(b) Period 2

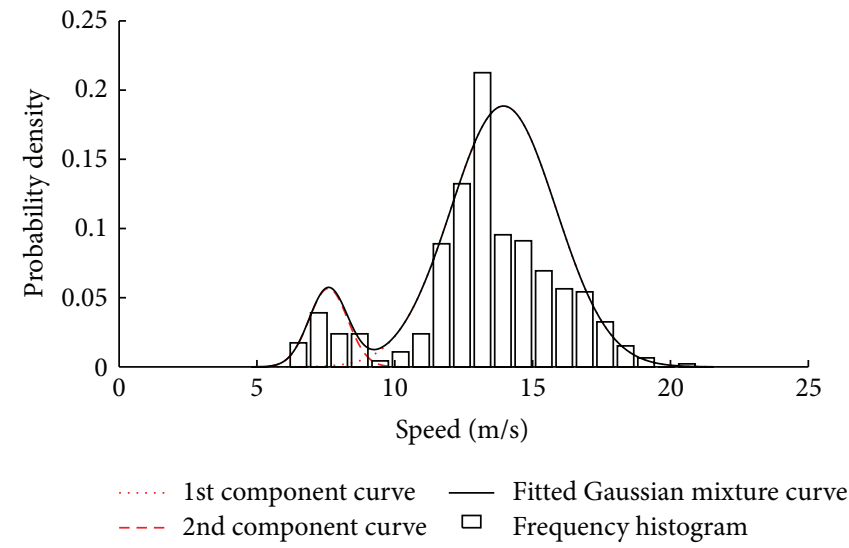

(c) Period 3

FIGURE 2: Speed distribution histogram and fitted Gaussian mixture distribution curve of the study segment.

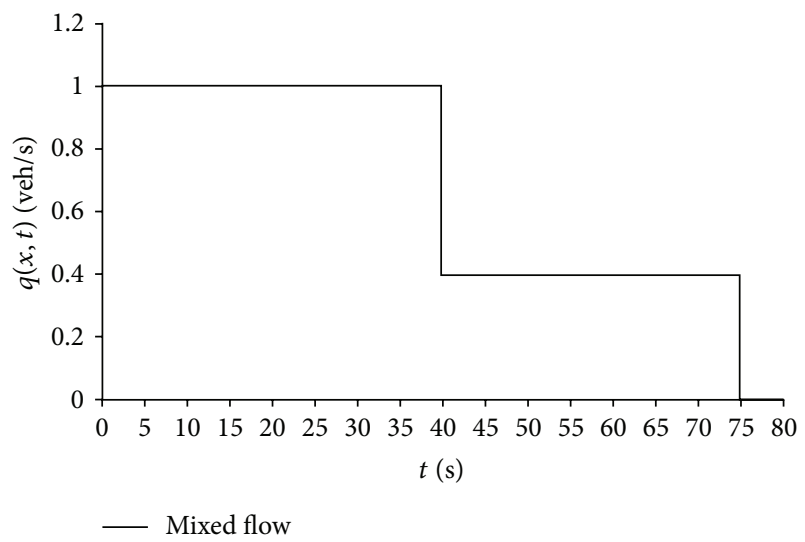

FIGURE 3: Departing flow distribution at upstream intersection.

numeric analysis and are shown in Figure 3. The mixed flow is the sum of car and bus flows.

4.2. Arriving Flow Distribution at Downstream Intersection. The virtual downstream intersections are assumed at $x_{d}=$ $100,400,700(\mathrm{~m})$. The arriving flow distribution function $q\left(x=x_{d}, t\right)$ is analyzed for mixed platoon. Because

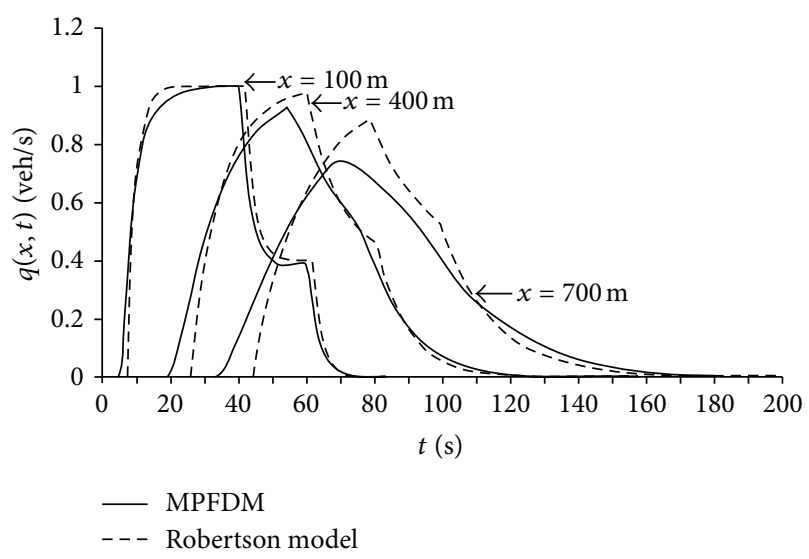

Figure 4: Comparison of arriving flow distribution between the proposed model and the Robertson model.

the Robertson model is widely known, its details are not presented here.

The arriving flow distribution at downstream locations at $x_{d}=100,400,700(\mathrm{~m})$ for mixed platoon using different modeling methods is presented in Figure 4.

Based on Figure 4, the following can be concluded regarding the model performance. 
TABLE 3: Statistics of TGMD of Time Period 1.

\begin{tabular}{lcc}
\hline & Symbol & Mixed platoon \\
\hline TGMD coefficient & $c$ & 1.055 \\
Minimum speed $(\mathrm{m} / \mathrm{s})$ & $\nu_{\min }$ & 5.65 \\
Maximum speed $(\mathrm{m} / \mathrm{s})$ & $\nu_{\max }$ & 20.97 \\
& $\alpha_{1}$ & 0.8290 \\
Parameters of Gaussian & $\alpha_{2}$ & 0.1710 \\
mixture distribution & $\mu_{1}$ & 13.6642 \\
& $\mu_{2}$ & 8.9297 \\
& $\sigma_{1}$ & 3.2344 \\
& $\sigma_{2}$ & 4.0870 \\
\hline
\end{tabular}

(a) According to MPFDM, during time period $\forall t \notin$ $\left[x_{d} / v_{\max }, x_{d} / v_{\min }+g\right]$, the flow rate $q\left(x_{d}, t\right)=$ 0 ; when $x / v_{\min } \leq x / v_{\max }+g$, during time period $\forall t \in\left[x_{d} / v_{\max }, x_{d} / v_{\text {min }}+g\right]$, the flow rate $q\left(x_{d}, t\right)$ increases as $t$ increases; during time period $\forall t \in\left[x_{d} / v_{\min }, x_{d} / v_{\min }+g\right]$, the flow rate $q\left(x_{d}, t\right)$ starts to decrease; when $x / v_{\min }>x / v_{\max }+$ $g$, during time period $\forall t \in\left[x_{d} / v_{\text {max }}, x_{d} / v_{\text {min }}\right]$, the flow rate $q\left(x_{d}, t\right)$ increases as $t$ increases; during time period $\forall t \in\left[x_{d} / v_{\min }, x_{d} / v_{\min }+g\right]$, the flow rate $q\left(x_{d}, t\right)$ starts to decrease. Furthermore, as $x_{d}$ increases, the peak flow rate decreases, and it will take longer time $x_{d} / v_{\min }+g-x_{d} / v_{\max }$ for all vehicles to pass the downstream intersection. This is as observed in the actual world. However, the Robertson model lacks the capability of modeling this phenomenon.

(b) Compared to the Robertson model, vehicles at the front of the platoon reach the downstream intersection earlier and those at the rear of platoon spread in a shorter range for MPFDM. As the distance increases, the difference increases. This is because the platoon speed of MPFDM follows TGMD, which spreads in a narrower range in $\left[v_{\min }, v_{\max }\right]$.

(c) Compared to the Robertson model, the peak of flow is lower and appears as a smooth hump for MPFDM, and the hump becomes flatter as the distance increases. This is due to faster vehicles presented in the Robertson model and the fact that the volume conservation rule cannot be violated.

(d) Compared to the Robertson model, MPFDM presents the exact time the first vehicle and the last vehicle reaches the downstream intersection, which also reflects the fact in the field. However, vehicles travelling at a very small or even zero speed exist in the Robertson model.

\section{Conclusion}

Large percentage of bus flow in mixed flow affects the accuracy of platoon dispersion modeling in Pacey's model or the Robertson model, which does not discriminate between bus traffic and car traffic. Through speed TGMD assumption, the mixed flow can be modeled by combining bus platoon with car platoon. Mixed platoon speed distribution will be influenced by the interaction between cars and buses, which is affected by flow rate, roadway function class, and percentage of buses. However, the interaction will eventually manifest a complicated speed distribution which cannot deal with simple distribution [27].

This strategy used here for mixed platoon modeling can be applied for all kinds of vehicle-type combination. For buscar mixed traffic, only this mixed platoon dispersion model is needed; for multiple vehicle types, because platoon speed distribution can be fitted by adjusting the number of mixed components, the arriving mixed flow at the downstream intersection can be obtained. Therefore, the model has wide application value.

Vehicles with infinite speeds exist in both Pacey's model and Robertson model, which violate the speed distribution limits (minimum and maximum speeds) in the actual world. The proposed truncated distribution assumption fixes the defect of those models.

\section{Acknowledgments}

This work was supported by the National Science Foundation of China under Contract no. 61174188. This support is gratefully acknowledged. The authors also thank Dr. Shen for his instructive advice and useful suggestion.

\section{References}

[1] G. M. Pacey, “The progress of a bunch of vehicles released from a traffic signal," Research Note No. RN/2665/GMP, Road Research Laboraroty, Berkshire, UK, 1956.

[2] M. J. Grace and R. B. Potts, "A theory of the diffusion of traffic platoons," Operation Research, vol. 12, pp. 255-275, 1964.

[3] D. I. Robertson, “TRANSYT-a network study tool," RRL Report LR 253, Road Research Laboratory, Berkshire, UK, 1969.

[4] J. A. Hillier and R. W. Rothery, "The synchronization of traffic signals for minimum delay," Transportation Science, vol. 1, pp. 81-93, 1967.

[5] “TRANSYT-7F User's Manual Release 10 (TM),” 2006.

[6] P. B. Hunt, F. I. Robertson, R. D. Bretherton, and R. I. Winton, "SCOOT-a traffic responsive method of coordinating signals, RRL tool," RRL Report LR 1041, Road Research Laboratory, Berkshire, UK, 1981.

[7] M. D. Hall, D. van Vliet, and L. G. Willumsen, "SATURNa simulation/assignment model for the evaluation of traffic management schemes," Traffic Engineering and Control, vol. 21, no. 4, pp. 168-176, 1980.

[8] E. B. Lieberman and B. J. Andrews, "TRAFLO-a new tool to evaluate transportation management strategies," Transportation Research Record, vol. 772, pp. 9-15, 1980, TRB, National Research Council, Washington, DC, USA.

[9] P. A. Seddon, "Another look at platoon dispersion: 3. The recurrence relationship," Traffic Engineering and Control, vol. 13, no. 10, pp. 442-444, 1972.

[10] M. Tracz, "The prediction of platoon dispersion based on rectangular distribution of journey time," Traffic Engineering and Control, vol. 16, no. 11, pp. 490-492, 1975.

[11] A. Polus, "A study of travel time and reliability on arterial routes," Transportation, vol. 8, no. 2, pp. 141-151, 1979. 
[12] C. Liu and P. Yang, "Diffusion models of traffic platoon on signal-intersection and control of coordinated signals," Journal of Tongji University (Nature Science Edition), vol. 24, no. 6, pp. 636-641, 1996.

[13] C. Q. Liu and P. K. Yang, "Diffusion model of density of traffic platoon and signal coordinated control," China Journal of Highway and Transport, vol. 14, no. 1, pp. 89-91, 2001.

[14] C. Q. Liu and P. K. Yang, "Modification of Grace's density diffusion model and its application," Journal of Highway and Transportation Research and Development, vol. 18, no. 1, pp. 6264, 2001.

[15] D. H. Wang, F. Li, and X. M. Song, "A new platoon dispersion model and its application," Journal of Jilin University (Engineering and Technology Edition), vol. 39, no. 4, pp. 891-896, 2009.

[16] D. Wang, Y. Zhang, and Z. Wang, "Study of platoon dispersion models," in Proceedings of the TRB Annual Meeting, Washington, DC, USA, 2003.

[17] M. Wei, W. Jin, and L. Shen, "A platoon dispersion model based on a truncated normal distribution of speed," Journal of Applied Mathematics, vol. 2012, Article ID 727839, 13 pages, 2012.

[18] A. Manar and K. G. Baass, "Traffic platoon dispersion modeling on arterial streets," Transportation Research Record, vol. 1566, pp. 49-53, 1996.

[19] G. C. K. Wong and S. C. Wong, "A multi-class traffic flow model-an extension of LWR model with heterogeneous drivers," Transportation Research A, vol. 36, no. 9, pp. 827-841, 2002.

[20] J. A. Bonneson, M. P. Pratt, and M. A. Vandehey, "Predicting arrival flow profiles and platoon dispersion for urban street segments," Transportation Research Record, vol. 2173, pp. 28-35, 2010.

[21] X. Cheng, "Distribution of vehicle free flow speeds based on Gaussian mixture model," Journal of Highway and Transportation Research and Development, vol. 29, no. 8, pp. 132-135, 2012.

[22] J. Chen, T. Wang, C. Li, and C. Yuan, "Speed models of mixed traffic flow on bus-car and vehicle and analysis of traffic running state," China Journal of Highway and Transport, vol. 25, no. 1, 2012.

[23] L. J. Yu, "Finite weibull mixture distribution model of VOT distribution forecasting," China Journal of Highway and Transport, vol. 22, no. 4, pp. 96-101, 2009.

[24] P. Tao, D. Wang, and S. Jin, "Mixed distribution model of vehicle headway," Journal of Southwest Jiaotong University, vol. 46, no. 4, pp. 633-637, 2011.

[25] A. D. May, Traffic Flow Fundamentals, Prentice Hall, New York, NY, USA, 1990.

[26] N. H. Johnson and S. Kotz, Continuous Univariate Distributions, John Wiley \& Sons, New York, NY, USA, 1970.

[27] Transit Capacity and Quality of Service Manual, Transportation Research Board, Washington, DC, USA, 2nd edition, 2003. 


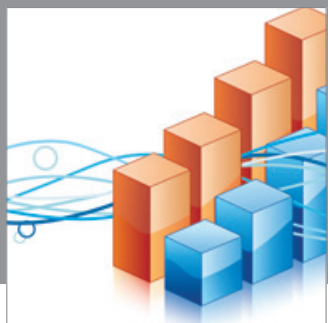

Advances in

Operations Research

mansans

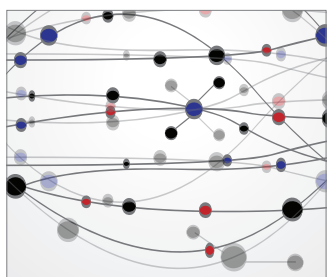

The Scientific World Journal
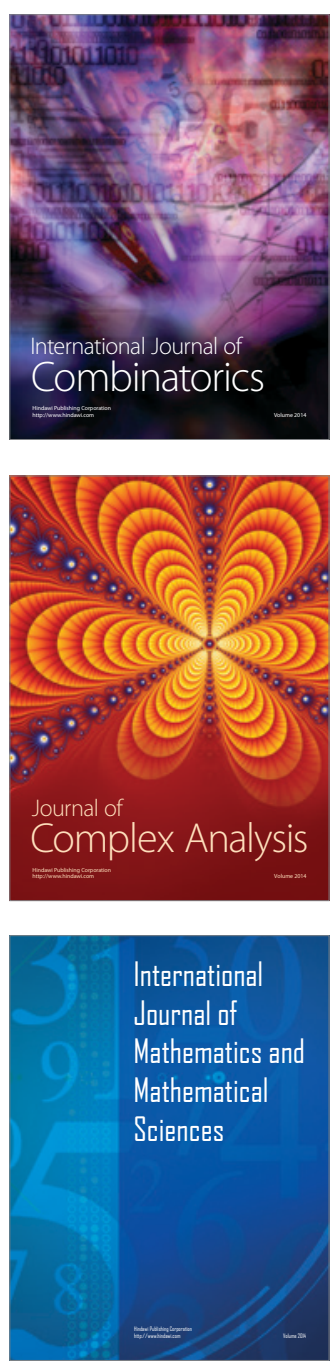
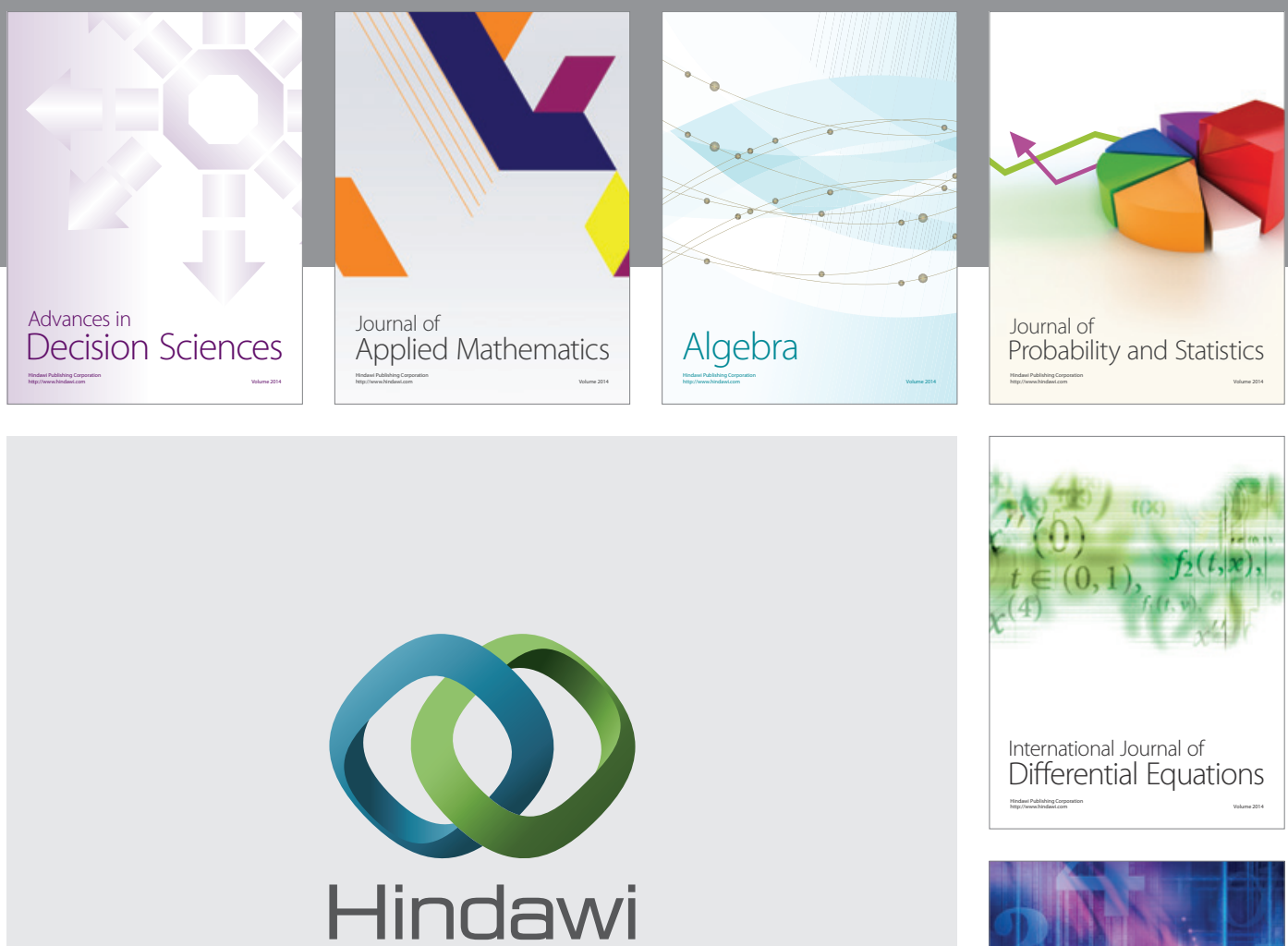

Submit your manuscripts at http://www.hindawi.com
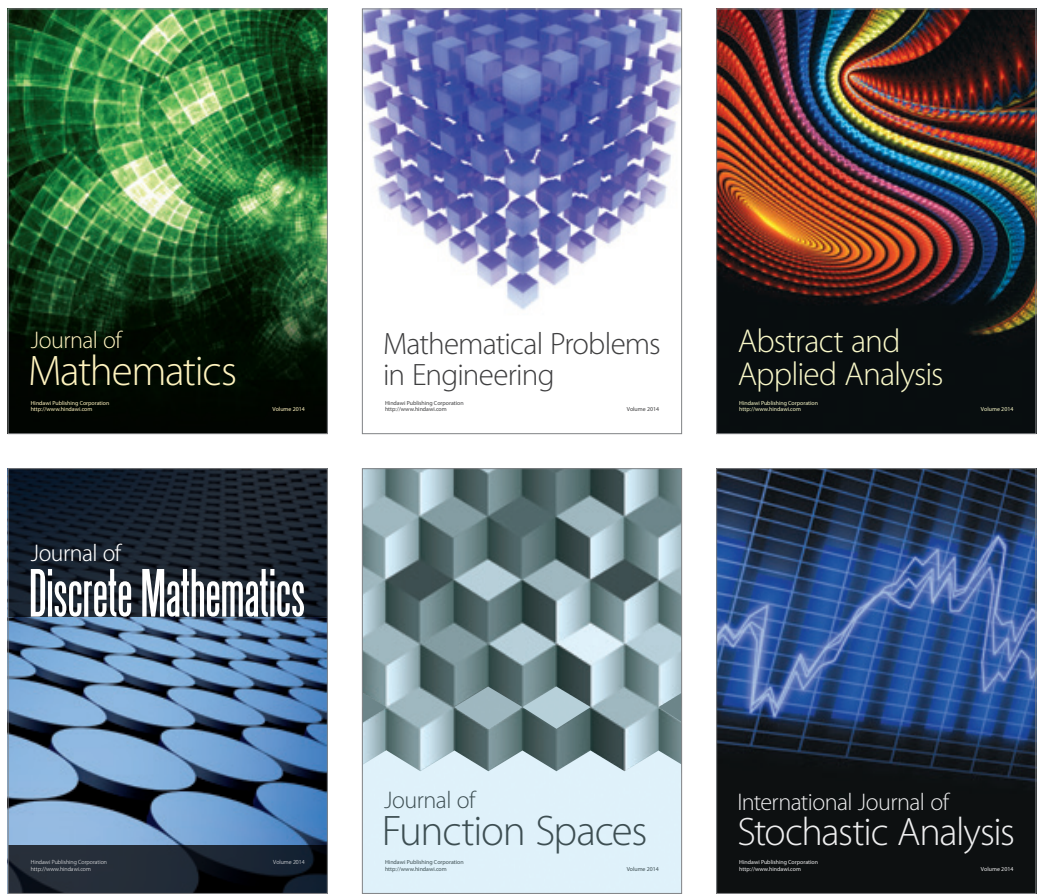

Journal of

Function Spaces

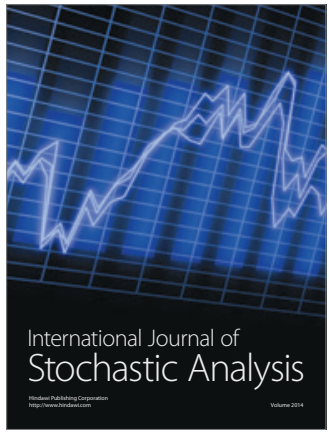

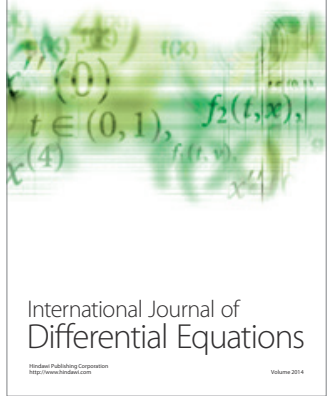
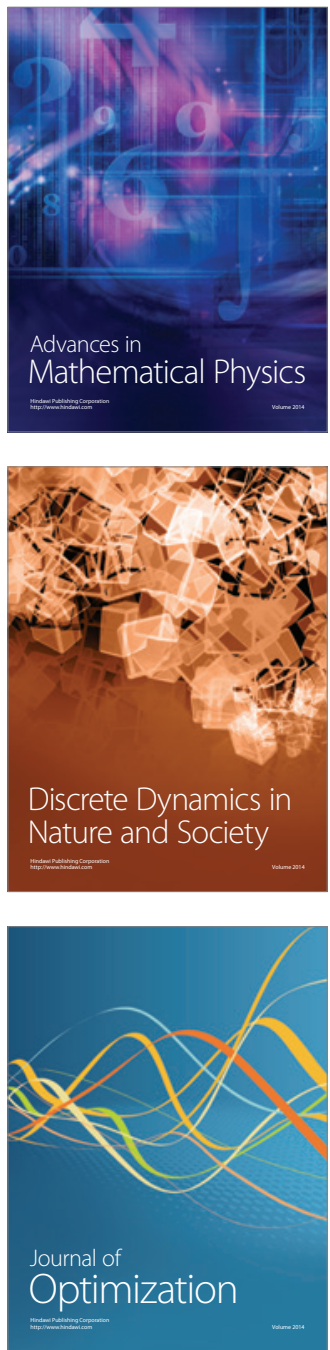